\title{
Level-triggered Control of a Scalar Linear System
}

\author{
Maben Rabi \\ Automatic Control Laboratory \\ School of Electrical Engineering \\ Royal Institute of Technology \\ Stockholm 100 44, Sweden \\ Email: mabenr@ee.kth.se
}

\author{
John S. Baras \\ Institute for Systems Research and \\ Department of Electrical and Computer Engineering \\ University of Maryland \\ College Park, MD 20742. USA \\ Email: baras@isr.umd.edu
}

\begin{abstract}
In networked control systems, because of limits on communication rates, control waveforms are frequently piece-wise constant with limits on rates at which they can switch levels. It is, therefore, natural to expect eventtriggered control switchings to perform more efficiently than time-triggered ones. This article verifies the validity of this supposition. A method for designing good level-triggered control schemes is obtained by reducing the continuous time problem to one in discrete time. Then, by numerical procedures, the performance of the level-triggered scheme is computed for comparison with that of the periodically switched control scheme.
\end{abstract}

\section{EVENT-TRIGGERED CONTROL FOR NETWORKED SYSTEMS}

Event-triggered controls have been shown in some settings to be more efficient than time-triggered ones. The paper [1] treats a problem of minimum variance stabilization of a scalar linear plant using state-resetting impulse controls. It provides a comparison of periodic impulse control and impulse control triggered by $\Delta$ excursions of the state from the origin. By explicit calculations, it is shown [1] that for the same average rate of impulse invocation, the level-triggered scheme provides lower average stabilization error.

This article provides a similar comparison when the control is not of impulse type, but is piecewise continuous. In fact the class of control signals considered is piecewise constant, these being natural candidates for situations in Networked Control. There, the control signals can change values no more than a prescribed average number of times. The control task is to obtain good stabilization performance while not exceeding the limits on average switching rates. The analysis and design of these setups is more involved than that of impulse control. So, we are only able to provide a comparison by numerical computations.

We show that level-triggered control switching schemes are superior to periodically switched control schemes. We furnish an important step, which is needed in order to be able to design good level-triggered schemes. This concerns a reduction of the continuous time event-triggered

This research was supported by the Army Research Office under the ODDR\&E MURI01 Program Grant No. DAAD19-01-1-0465 to the Center for Networked Communicating Control Systems (through Boston University), and under ARO Grant No. DAAD190210319. control problem to an equivalent control problem for a discrete time Markov chain. While this is analogous to the development for periodic switching of control signals, the resulting discrete-time average cost control problem does not have an explicitly computable solution. This is why, the performance comparison has to be computed numerically.

In the following section, we discuss how, in a basic Networked Control setting, by assuming reliable and delayfree delivery of data packets, event-triggered sampling of state observations leads to an event-triggered control problem.

\section{Average Cost Control Problem}

Consider a single sensor configuration depicted in Figure 1.

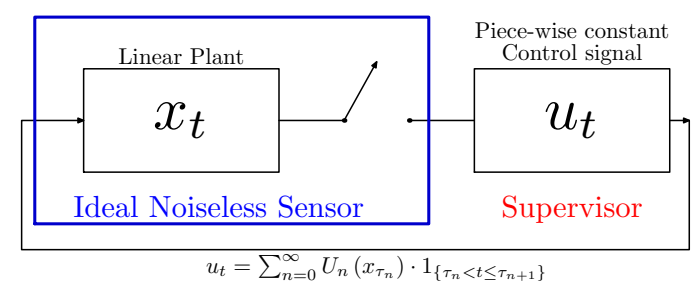

Fig. 1. Sample and hold control

We have a scalar state signal which is a controlled linear system. On an infinite horizon, the sensor sends samples to the supervisor which issues a piece-wise constant control signal. The values of the control signal are allowed to change only at times when the supervisor receives samples. The control objective is to minimize the average power of the state signal. The sensor's objective is to aid in the control task and send samples as often as it can while also respecting a bound on the average sampling rate.

The state signal obeys:

$$
d x_{t}=a x_{t} d t+d W_{t}+u_{t} d t, \quad x(0)=x_{0},
$$

where $W_{t}$ is a standard Brownian motion process and the control signal is piecewise constant and adapted to the sampled stream. Let $\mathcal{T}$ be the sequence of sampling times:

$$
\mathcal{T}=\left\{\tau_{0}, \tau_{1}, \tau_{2}, \ldots\right\}
$$


with

$$
\begin{aligned}
& \tau_{0}=0, \\
& \tau_{i}<\tau_{i+1} \text { a.s. for } i \geq 0, \text { and } \\
& \tau_{i} \text { is measurable w.r.t. } \mathcal{F}_{t}^{x} .
\end{aligned}
$$

Then the (stationary feedback) control policy $U$ should be adapted to the sample sequence. Define the process $\left\{\Psi_{t}\right\}$ describing information available at the controller from the last received data packet:

$$
\Psi_{t}=\left(\begin{array}{c}
\chi_{t} \\
\nu_{t}
\end{array}\right)=\left(\begin{array}{c}
\sum_{n=0}^{\infty} 1_{\left\{\tau_{n}<t \leq \tau_{n+1}\right\}} \cdot x_{\tau_{n}} \\
\sum_{n=0}^{\infty} 1_{\left\{\tau_{n}<t \leq \tau_{n+1}\right\}} \cdot \tau_{n}
\end{array}\right) .
$$

Let $\mathscr{U}$ stand for the set of control policies $U$ that are adapted to the $\Psi$-process. The actual control signal generated by $U$ is given by:

$$
u_{t}=\sum_{n=0}^{\infty} 1_{\left\{\tau_{n}<t \leq \tau_{n+1}\right\}} \cdot U_{n}\left(x_{\tau_{n}}\right),
$$

where $U_{n}\left(x_{\tau_{n}}\right)$ is the value of the control signal after the sample at time $\tau_{n}$ has been received.

Stabilization performance is measured through the average power of the state signal:

$$
J_{u}=\limsup _{T \rightarrow \infty} \frac{1}{T} \mathbb{E}\left[\int_{0}^{T} x_{s}^{2} d s\right],
$$

while the average sampling rate:

$$
R=\limsup _{T \rightarrow \infty} \frac{1}{T} \mathbb{E}\left[\int_{0}^{T} \sum_{n=0}^{\infty} \mathbf{1}_{\left\{\tau_{n} \leq T\right\}} \delta\left(s-\tau_{n}\right) d s\right]
$$

is kept less than or equal to a desired bound. Here, $\delta(\cdot)$ is the Dirac-delta function.

Since we use stationary feedback controls, the sampled stream forms a controlled Markov chain in discrete-time. We will translate the continuous-time optimal control problem into an equivalent one in discrete time for which we seek solutions. In section III, we do this for the case of periodic sampling.

This problem differs in its information pattern from similar ones addressed in the Stochastic Control literature. The works [2]-[4] seek combined control and stopping policies with both of these adapted to the same signal process. In our problem on the other hand, the stopping policy is allowed to depend on the $x$-signal process while the control signal is adapted to the sampled sequence. The work of [5] discusses the LQG control performance under Poisson sampling. A deterministic version of control for event-triggered sampling is presented in [6]. A sensor scheduling problem related to our problem of sampling and control is treated in [7]. There, it is shown that sensor selection triggered by level crossings of an error process is optimal.

The problem of choosing jointly the event-triggered sampling matching control is a switching control problem with no known solution. Hence in this work, we will seek optimal control policies corresponding to a chosen sampling strategy. We will study the performance of the optimal controls corresponding to two types of leveltriggered sampling strategies described in section V.

\section{Optimal Control Under Periodic SAMPling}

Under periodic sampling, the sample times are given by

$$
\tau_{n}=n \Delta \quad \text { for } n \geq 0 .
$$

The sampled state takes the form of a discrete time linear system:

$$
\begin{aligned}
X_{n+1}= & e^{a T} X_{n}+\int_{n \Delta}^{(n+1) \Delta} e^{a((n+1) \Delta-s)} d W_{s} \\
& +\int_{n \Delta}^{(n+1) \Delta} e^{a((n+1) \Delta-s)} u_{s} d s \\
= & e^{a T} X_{n}+\sqrt{\frac{\mu^{2}-1}{2 a}} V_{n}+\frac{\mu-1}{a} U_{n},
\end{aligned}
$$

where $\left\{V_{n}\right\}$ is an IID sequence of standard Gaussian random variables and $\mu=e^{a \Delta}$.

It is easy to find feedback control policies $\left\{U_{n}\right\}$ that stabilize (in the mean square sense) the sampled linear system. For example, linear feedback controls of the form

$$
U_{n}=k X_{n}, \quad n \geq 0,
$$

with $-1<k+\frac{\mu^{2}-1}{2 a}<1$ will stabilize $\left\{X_{n}\right\}$. It can also be seen that stability of the sampled state sequence implies stability of the original continuous time system. We shall restrict our attention to mean-square stabilizing control policies that also make the controlled process (5) ergodic with a p.d.f such that the fourth moment at steady state is finite. We will need this restriction to translate the continuous time optimization problem into an equivalent one in discrete time. The class of linear feedback policies which are described above are included in our restricted policy space. Let $\rho_{U}$ be the steady state p.d.f. corresponding to the control policy $\left\{U_{n}\right\}$. When the sampled state sequence is ergodic, so is the actual continuous time state waveform.

\section{A. Equivalent Discrete Time Ergodic Control Problem}

The expected integral cost of section II, (2), is the sum of expected integrals over the inter-sample intervals. We want to be able to write the expected integral costs during such intervals as functions of the state of the chain at the beginning (or end) of the interval instead of being functions of the chain states at both end-points.

Because of the assumed ergodicity, we can replace the average cost (2) with the long run average cost (ergodic cost). Then, along the lines of lemma 3.4 of [8], we have:

$$
\begin{aligned}
J_{u} \stackrel{\text { a.s. }}{=} & \limsup _{T \rightarrow \infty} \frac{1}{T} \int_{0}^{T} x_{s}^{2} d s \\
= & \limsup _{N \rightarrow \infty} \frac{1}{N \Delta} \sum_{n=0}^{N-1} \int_{\tau_{n}}^{\tau_{n+1}} x_{s}^{2} d s \\
= & \limsup _{N \rightarrow \infty} \frac{1}{N \Delta} \sum_{n=0}^{N-1}\left\{\int_{\tau_{n}}^{\tau_{n+1}} x_{s}^{2} d s-\mathbb{E}\left[\int_{\tau_{n}}^{\tau_{n+1}} x_{s}^{2} d s \mid X_{n}\right]\right\} \\
& +\limsup _{N \rightarrow \infty} \frac{1}{N \Delta} \sum_{n=0}^{N-1} \mathbb{E}\left[\int_{\tau_{n}}^{\tau_{n+1}} x_{s}^{2} d s \mid X_{n}\right] .
\end{aligned}
$$


The first part of the last expression is zero according to the Martingale stability theorem (page 105 of [9]). We are able to use this theorem because of the finiteness of the fourth moment of the state signal.

Let $\delta(\cdot)$ denote the Dirac-delta function. We have:

$$
\begin{aligned}
J_{u} & \stackrel{\text { a.s. }}{=} \limsup _{N \rightarrow \infty} \frac{1}{N \Delta} \sum_{n=0}^{N-1} \mathbb{E}\left[\int_{\tau_{n}}^{\tau_{n+1}} x_{s}^{2} d s \mid X_{n}\right] \\
& =\limsup _{N \rightarrow \infty} \frac{1}{N \Delta} \sum_{n=0}^{N-1} \int_{\mathbb{R}} \mathbb{E}\left[\int_{\tau_{n}}^{\tau_{n+1}} x_{s}^{2} d s\right] \delta\left(x-X_{n}\right) d x \\
& =\frac{1}{\Delta} \int_{\mathbb{R}} \mathbb{E}\left[\int_{0}^{\Delta} x_{s}^{2} d s \mid X_{0}=x\right] \rho_{U}(x) d x \\
& =\frac{1}{\Delta} \int_{\mathbb{R}}\left\{A X_{n}^{2}+2 B X_{n} U_{n}+C U_{n}^{2}\right\} \rho_{U}(x) d x,
\end{aligned}
$$

where,

$$
\begin{aligned}
& A=\frac{\mu^{2}-1}{2 a}, \\
& B=\frac{1}{a}\left\{\frac{\mu^{2}-1}{2 a}-\frac{\mu-1}{a}\right\}, \\
& C=\frac{1}{a^{2}}\left\{\frac{\mu^{2}-1}{2 a}-2 \frac{\mu-1}{a}+T\right\} .
\end{aligned}
$$

In fact, we can write:

$$
\begin{aligned}
J_{u} & \stackrel{\text { a.s. }}{=} \limsup _{N \rightarrow \infty} \frac{1}{N \Delta} \sum_{n=0}^{N-1} A X_{n}^{2}+2 B X_{n} U_{n}+C U_{n}^{2} \\
& \triangleq G_{U} .
\end{aligned}
$$

The solution to this fully observed, average cost (which is identically equivalent to ergodic cost) control problem is well known [10]. The optimal controls are linear feedback controls. To find the optimal feedback gain, we use the Average Cost optimality equation:

$$
\begin{aligned}
\alpha^{*}+h(x)=\inf _{u \in \mathbb{R}}\left\{A x^{2}+2 B u x+C u^{2}\right. \\
\left.+\mathbb{E}\left[h\left(\mu x+\frac{\mu-1}{a} u+\sqrt{\frac{\mu^{2}-1}{2 a}} W_{0}\right)\right]\right\} .
\end{aligned}
$$

Here, $\alpha^{*}$ is the minimum cost and a quadratic choice for $h(\cdot)$ verifies the optimality of linear feedback. The optimal control is given by:

$$
U_{n}^{*}=-\frac{a\left(\mu^{2}-1\right)^{2}+2 a^{2} \theta \mu(\mu-1)}{(\mu-2)^{2}-1+2 a \Delta+2 a \theta(\mu-1)^{2}} X_{n},
$$

where,

$$
\begin{aligned}
\theta & =\frac{D}{2}+\sqrt{\frac{D^{2}}{2}+\frac{D}{2 a}} \\
D & =\frac{\mu+1}{\mu-1}\left(\frac{\mu^{2}-1}{2 a}-2 \frac{\mu-1}{a}+\Delta\right)-\frac{(\mu-1)^{2}}{2 a} .
\end{aligned}
$$

The minimum average cost is:

$$
\alpha^{*}=\frac{\theta}{\Delta} \frac{\left(\mu^{2}-1\right)^{2}}{2 a} .
$$

The sampling rate is of course equal to $\frac{1}{\Delta}$.

\section{LEVEL-TRIGGERED SAMPLING}

Let $\mathcal{L}$ be a given infinite set of levels:

$$
\begin{array}{r}
\mathcal{L}=\left\{\ldots, l_{-2}, l_{-1}, l_{0}, l_{1}, l_{2}, \ldots\right\}, \\
\text { with, } \quad \begin{cases}l_{i} \in \mathbb{R} & \forall i \in \mathbb{Z}, \\
l_{i}<l_{i+1} & \forall i \in \mathbb{Z}, \\
l_{0}=0 .\end{cases}
\end{array}
$$

If we want a set of levels all non-zero, we just remove $l_{0}$ from the set $\mathcal{L}$. We need an infinite set in order to be able to stabilize unstable plants.
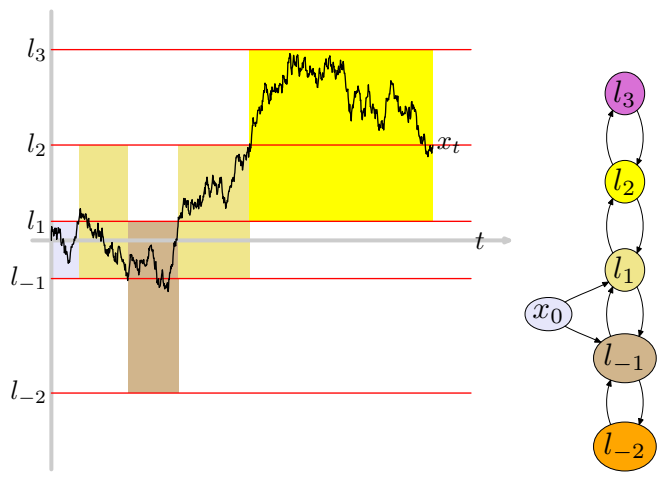

Fig. 2. Level-triggered sampling and the associated Markov chain. All levels are non-zero. The initial state does not belong to the set of levels $\mathcal{L}$. This gives rise to the only transient state ' $x_{0}$ '.

The sampling times triggered by $\mathcal{L}$ are defined through fresh crossings of levels:

$$
\begin{aligned}
& \tau=0, \\
& \tau=\inf \left\{\tau \mid \tau>\tau_{i}, x_{\tau} \in \mathcal{L}, x_{\tau} \notin x_{\tau_{i}}\right\} .
\end{aligned}
$$

We have to use fresh crossings instead of arbitrary crossings to keep the sampling rate finite. The expected intersample times depend on the state at the beginning of the interval as well as the control policy. We shall assume that the levels in $\mathcal{L}$ as well as the control policy are such that the expected inter-sample times are finite and bounded away from zero. When the plant is unstable, this means that the levels in $\mathcal{L}$ go up to $\infty$ and $-\infty$.

\section{A. Equivalent Discrete-time Markov Chain}

As with periodic sampling, the sequence

$$
\left\{X_{n} \mid n=0,1,2, \ldots ; X_{n}=x_{\tau_{n}}\right\}
$$

forms a discrete-time controlled Markov chain (Figure 2). Here, it takes values in the finite set $\mathcal{L}$. As before, we will assume that the discrete-time control sequence $\left\{U_{n}\right\}$ is such that the resultant Markov chain is ergodic and also stable in the following sense:

$$
\mathbb{E}\left[h^{4}(X)\right]<\infty,
$$

where, $h: \mathcal{L} \rightarrow \mathbb{R}$ is defined by

$$
h(l)=|l| \text {. }
$$

Like in section III-A, we can express the average quantities for the continuous time problem in terms of the ones for a related discrete-time controlled Markov chain $\left\{x_{n}\right\}$. 
The average sampling rate is given by [8]:

$$
R_{\mathcal{L}, U}=\frac{1}{\sum_{l_{i} \in \mathcal{L}} \pi_{U}\left(l_{i}\right) \zeta\left(l_{i}, U\left(l_{i}\right)\right)},
$$

where, $\zeta: \mathcal{L} \times \mathbb{R} \rightarrow \mathbb{R}^{+}$is the expected first exit time defined by:

$$
\zeta\left(l_{i}, U\left(l_{i}\right)\right)=\mathbb{E}\left[\tau_{1} \mid \tau_{0}=0, x_{0}=l_{i} \in \mathcal{L}, U_{0}=U\left(l_{i}\right)\right],
$$

and $\left\{\pi_{U}\left(l_{i}\right)\right\}_{i=-\infty}^{\infty}$ is the steady state distribution for $\left\{x_{n}\right\}$.

The average stabilization error is given by [8]:

$$
J_{\mathcal{L}, U}=\frac{\sum_{l_{i} \in \mathcal{L}} \pi_{U}\left(l_{i}\right) g\left(l_{i}, U\left(l_{i}\right)\right)}{\sum_{l_{i} \in \mathcal{L}} \pi_{U}\left(l_{i}\right) \zeta\left(l_{i}, U\left(l_{i}\right)\right)},
$$

where, $g: \mathcal{L} \times \mathbb{R} \rightarrow \mathbb{R}^{+}$is defined by:

$$
g(l)=\mathbb{E}\left[\int_{0}^{\tau_{1}} x_{s}^{2} d s \mid \tau_{0}=0, x_{0}=l_{i} \in \mathcal{L}, U_{0}=U\left(l_{i}\right)\right] .
$$

Both the numerator and the denominator in the last expression for the average stabilization error are dependent on $U$. But the denominator is just the reciprocal of the average sampling rate which is constrained. Define the cost $\widetilde{J}_{\mathcal{L}, U}$ by:

$$
\tilde{J}_{\mathcal{L}, U}=\sum_{l_{i} \in \mathcal{L}} \pi_{U}\left(l_{i}\right) g\left(l_{i}, U\left(l_{i}\right)\right) .
$$

Then, minimizing $J_{\mathcal{L}, U}$ while respecting the constraint on the average sampling rate is the same as minimizing

$$
\frac{\tilde{J}_{\mathcal{L}, U}}{\sum_{l_{i} \in \mathcal{L}} \pi_{U}\left(l_{i}\right) \zeta\left(l_{i}, U\left(l_{i}\right)\right)}
$$

while ensuring that

$$
R_{\mathcal{L}, U}=\frac{1}{\sum_{l_{i} \in \mathcal{L}} \pi_{U}\left(l_{i}\right) \zeta\left(l_{i}, U\left(l_{i}\right)\right)} \leq R_{\text {desired }}
$$

It is clear from the problem setup that under optimal $U$, both $\tilde{J}_{\mathcal{L}, U}$ and $R_{\mathcal{L}, U}$ are finite, positive and nonzero. If $\Gamma^{*}>0$ is the minimum value of $J_{\mathcal{L}, U}$, then, while respecting the constraint on average sampling rate (average inter-sample interval),

$$
\begin{aligned}
& \left\{\begin{array}{l}
\frac{\tilde{J}_{\mathcal{L}, U}}{\sum_{l_{i} \in \mathcal{L}} \pi_{U}\left(l_{i}\right) \zeta\left(l_{i}, U\left(l_{i}\right)\right)} \geq \Gamma^{*}>0, \quad \forall U \in \mathscr{U} \\
\frac{\tilde{J}_{\mathcal{L}, U^{*}}}{\sum_{l_{i} \in \mathcal{L}^{*}} \pi_{U^{*}}\left(l_{i}\right) \zeta\left(l_{i}, U^{*}\left(l_{i}\right)\right)}=\Gamma^{*} .
\end{array}\right. \\
& \Leftrightarrow\left\{\begin{array}{l}
\tilde{J}_{\mathcal{L}, U}-\Gamma^{*} \sum_{l_{i} \in \mathcal{L}} \pi_{U}\left(l_{i}\right) \zeta\left(l_{i}, U\left(l_{i}\right)\right) \geq 0, \quad \forall U \in \mathscr{U} \\
\tilde{J}_{\mathcal{L}, U^{*}}-\Gamma^{*} \sum_{l_{i} \in \mathcal{L}} \pi_{U^{*}}\left(l_{i}\right) \zeta\left(l_{i}, U^{*}\left(l_{i}\right)\right)=0 .
\end{array}\right.
\end{aligned}
$$

This means we only have to worry about minimizing $\tilde{J}_{\mathcal{L}, U}$ subject to the sampling rate constraint. This is the same as minimizing the Lagrangian:

$$
\tilde{J}_{\mathcal{L}, U}-\Gamma \sum_{l_{i} \in \mathcal{L}} \pi_{U}\left(l_{i}\right) \zeta\left(l_{i}, U\left(l_{i}\right)\right) .
$$

Denote the second sum in the above Lagrangian, the average inter-sample time, by $S_{\mathcal{L}, U}$.

We will now turn to the calculation of the transition probability kernel of $\left\{x_{n}\right\}$, and the average quantities $\tilde{J}_{\mathcal{L}, U}, S_{\mathcal{L}, U}$. To do so, we will appeal to the results of chapter 9 in [11]. Because the state signal is scalar, there are only two possible transitions from any state in $\mathcal{L}$. The transition probabilities

$$
\begin{array}{r}
p\left(l^{\prime}, l, U\right)=\mathbb{P}\left[X_{n+1}=l^{\prime} \mid X_{n}=l, U_{n}=U(l)\right], \\
\forall\left(l^{\prime}, l, U\right) \in \mathcal{L} \times \mathcal{L} \times \mathscr{U},
\end{array}
$$

are found by solving an ODE [11]:

$$
p\left(l_{i+1}, l_{i}, U\right)=\eta\left(l_{i}\right),
$$

where $\eta(\cdot)$ satisfies:

$$
(u+a x) \frac{d \eta}{d x}+\frac{1}{2} \frac{d^{2} \eta}{d x^{2}}=0,
$$

with the boundary conditions:

$$
\eta\left(l_{i+1}\right)=1, \eta\left(l_{i-1}\right)=0 .
$$

Then we have $\forall l_{i} \in \mathcal{L}$ :

$$
p\left(l, l_{i}, U\right)= \begin{cases}\frac{\int_{l_{i-1}}^{l_{i}} e^{-2 u s-a s^{2}} d s}{\int_{l_{i-1}}^{i+1} e^{-2 u r-a r^{2}} d r} & \text { if } l=l_{i+1}, \\ \int_{l_{i}}^{i+1} e^{-2 u s-a s^{2}} d s & \text { if } l=l_{i-1}, \\ \int_{l_{i-1}}^{i+1} e^{-2 u r-a r^{2}} d r & \text { otherwise. } \\ 0 & \end{cases}
$$

The steady-state occupation measure $\pi_{U}$ can be calculated using the equations below. For every $l_{i} \in \mathcal{L}$

$\pi_{U}\left(l_{i}\right)=\pi_{U}\left(l_{i-1}\right) p\left(l_{i-1}, l_{i}, U\right)+\pi_{U}\left(l_{i+1}\right) p\left(l_{i+1}, l_{i}, U\right)$

The expected stabilization error starting at level $l_{i}$ up to the first exit time out of $\left(l_{i-1}, l_{i+1}\right)$ is given by:

$$
g\left(l_{i}, U\right)=\eta\left(l_{i}\right),
$$

where $\eta(\cdot)$ satisfies the ODE:

$$
(u+a x) \frac{d \eta}{d x}+\frac{1}{2} \frac{d^{2} \eta}{d x^{2}}=-x^{2},
$$

with the boundary conditions:

$$
\eta\left(l_{i+1}\right)=0, \eta\left(l_{i-1}\right)=0 .
$$

Let

$$
\begin{aligned}
& q_{1}(x)=\int_{0}^{x} e^{-a z^{2}-2 u z} d z, \text { and, } \\
& q_{1}(x)=\int_{0}^{x} e^{-a z^{2}-2 u z} \int_{0}^{z} y^{2} e^{a y^{2}+2 u y} d y d z .
\end{aligned}
$$

Then we have $\forall l_{i} \in \mathcal{L}$ :

$$
\begin{aligned}
g\left(l_{i}, U\right)= & \frac{q_{1}\left(l_{i}\right) q_{2}\left(l_{i+1}\right)-q_{1}\left(l_{i}\right) q_{2}\left(l_{i-1}\right)-q_{1}\left(l_{i+1}\right) q_{2}\left(l_{i}\right)}{q_{1}\left(l_{i+1}\right)-q_{1}\left(l_{i-1}\right)} \\
& +\frac{q_{1}\left(l_{i-1}\right) q_{2}\left(l_{i}\right)-q_{1}\left(l_{i+1}\right) q_{2}\left(l_{i+1}\right)+q_{1}\left(l_{i+1}\right) q_{2}\left(l_{i-1}\right)}{q_{1}\left(l_{i+1}\right)-q_{1}\left(l_{i-1}\right)} .
\end{aligned}
$$

Similarly, The expected first exit time $\mathcal{E}\left(l_{i}, U\right)$ is given as below. We have $\forall l_{i} \in \mathcal{L}$ :

$$
\begin{aligned}
\mathcal{E}\left(l_{i}, U\right)= & \frac{q_{1}\left(l_{i}\right) q_{3}\left(l_{i+1}\right)-q_{1}\left(l_{i}\right) q_{3}\left(l_{i-1}\right)-q_{1}\left(l_{i+1}\right) q_{3}\left(l_{i}\right)}{q_{1}\left(l_{i+1}\right)-q_{1}\left(l_{i-1}\right)} \\
& +\frac{q_{1}\left(l_{i-1}\right) q_{3}\left(l_{i}\right)-q_{1}\left(l_{i+1}\right) q_{3}\left(l_{i+1}\right)+q_{1}\left(l_{i+1}\right) q_{3}\left(l_{i-1}\right)}{q_{1}\left(l_{i+1}\right)-q_{1}\left(l_{i-1}\right)} .
\end{aligned}
$$

where

$$
q_{3}(x)=\int_{0}^{x} e^{-a z^{2}-2 u z} \int_{0}^{z} e^{a y^{2}+2 u y} d y d z .
$$


As before, this expression follows from computing the expectation as the solution to a Dirichlet boundary value problem:

$$
\mathcal{E}\left(l_{i}, U\right)=\eta\left(l_{i}\right),
$$

where $\eta(\cdot)$ satisfies the ODE:

$$
(u+a x) \frac{d \eta}{d x}+\frac{1}{2} \frac{d^{2} \eta}{d x^{2}}=-1,
$$

with the boundary conditions:

$$
\eta\left(l_{i+1}\right)=0, \eta\left(l_{i-1}\right)=0 .
$$

\section{B. Existence of Optimal Controls and Their Computation}

The Markov chain $\left\{X_{n}\right\}$ has the property that, independent of $U$, only a finite number of elements of $\mathcal{L}$ can be reached from any member of $\mathcal{L}$ in one step. The per stage cost in the average cost formulation is an unbounded function of the state. For such situations, Borkar [12] shows the existence of optimal (non-randomized) stationary policies and proves the validity of the Average Cost optimality equations:

$$
\begin{aligned}
& \forall l_{i} \in \mathcal{L}, \quad \alpha^{*}=\inf _{u_{i} \in \mathbb{R}}\left\{g\left(l_{i}, u_{i}\right)-\Gamma \mathcal{E}\left(l_{i}, u_{i}\right)-v_{i}\right. \\
& \left.\quad+p\left(l_{i+1}, l_{i}, u_{i}\right) v_{i+1}+p\left(l_{i-1}, l_{i}, u_{i}\right) v_{i-1}\right\}
\end{aligned}
$$

We use value iteration based on the above equations to determine the optimal controls and their performance for fixed $\mathcal{L}$. We will next consider some natural classes of level-triggered sampling schemes.

\section{COMPARISONS}

We will consider two level-triggered sampling schemes. One will be the Lattice-triggered sampling scheme. Let

$$
\text { Latt }_{0}=\{\ldots,-2 \kappa,-\kappa, 0, \kappa, 2 \kappa, \ldots\} .
$$

Choosing $\mathcal{L}$ to be be Latt $_{0}$ gives a set of equi-spaced levels. Choosing $\mathcal{L}$ to be

$$
\text { Latt }_{1}=\{\ldots,-2 \kappa,-\kappa, \kappa, 2 \kappa, \ldots\},
$$

which does not have zero as a level leads to a variant of the equi-spaced set.

On the other hand, choosing $\mathcal{L}$ to be

$$
\begin{array}{r}
\log _{0}=\{\ldots,-\log (1+2 \kappa),-\log (1+\kappa), 0, \\
\log (1+\kappa), \log (1+2 \kappa), \ldots\}
\end{array}
$$

gives us a logarithmic set of levels and choosing $\mathcal{L}$ to be

$$
\begin{aligned}
& \log _{1}=\{\ldots,-\log (1+2 \kappa),-\log (1+\kappa), \\
&\log (1+\kappa), \log (1+2 \kappa), \ldots\}
\end{aligned}
$$

gives us a variant.

In Figures 3, 4, 5 and 6, we have sketched the performances of level-triggered schemes with these levels as well as the periodic sampling scheme.

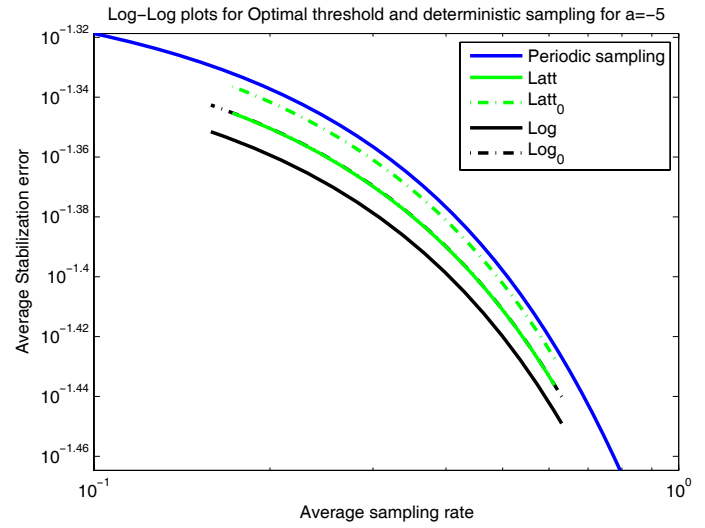

Fig. 3. Relative performance of Threshold and Periodic sampling for $a=-5$.

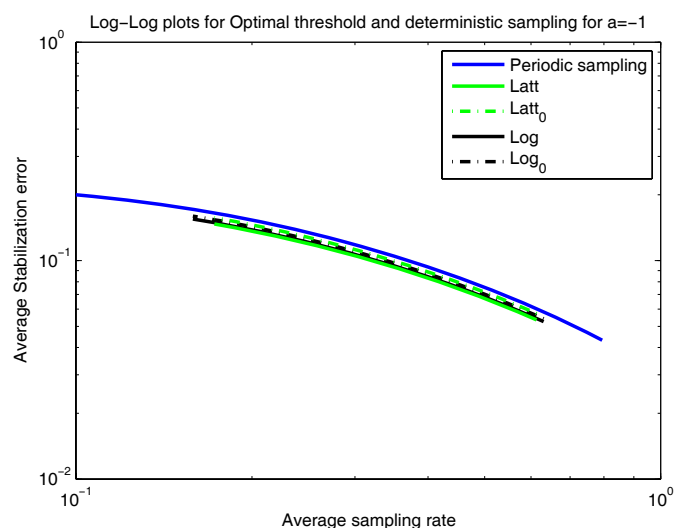

Fig. 4. Relative performance of Threshold and Periodic sampling for $a=-1$.

In the optimal control problems dealt so far, we could relax the restriction to controls that render the system ergodic with a finite steady state fourth moment. But doing so forces us to modify the state space. The new Markov chain to be worked with has the state:

$$
Z_{n}=\left(\begin{array}{c}
X_{n} \\
Y_{n}
\end{array}\right)=\left(\begin{array}{c}
X_{n} \\
X_{n-1}
\end{array}\right), \quad \forall n \geq 1, \quad S_{0}=\left(\begin{array}{c}
X_{0} \\
x
\end{array}\right),
$$

where, $x$ is any valid element of the chain's state space that can have $X_{0}$ as a successor state. Now the expected integral running cost over the inter-sample interval $\left[\tau_{n-1}, \tau_{n}\right)$ is purely a function of $Z_{n}$. However, the computation of the parameters of the Markov chain and the solution of the average cost control problem are more involved.

\section{CONCluding Remarks}

We have solved an average cost feedback control problem with reliable delivery of samples. We need to find ways of obtaining the optimal set of levels for eventtriggered sampling. We need to see how the performances of the various sampling schemes compare when the signal is nonlinear. Extension to the case of a vector signal is non-trivial.

On the other hand, using multiple sensors for estimating a scalar state signal leads to a tractable analysis of leveltriggered sampling. We could sample when the local 


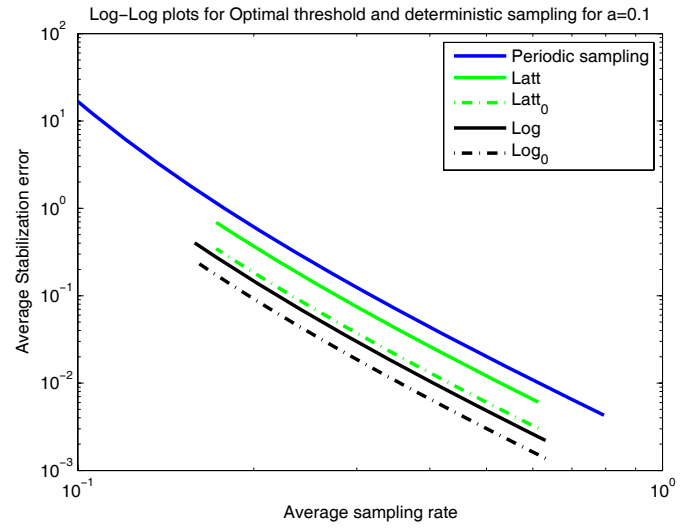

Fig. 5. Relative performance of Threshold and Periodic sampling for $a=0.1$

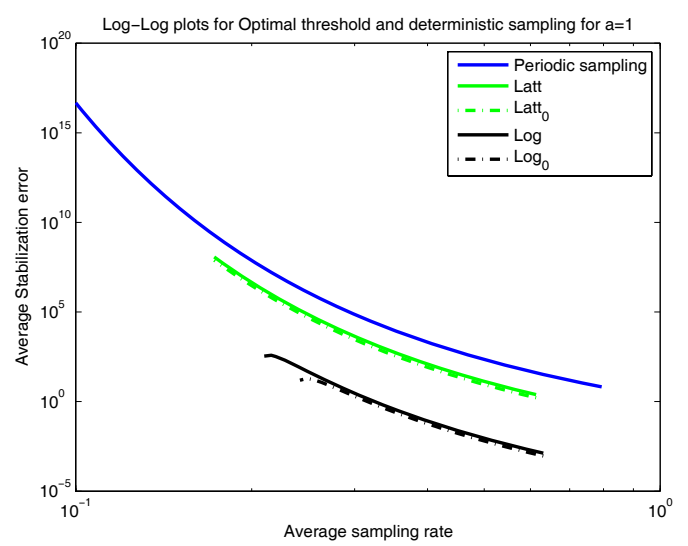

Fig. 6. Relative performance of Threshold and Periodic sampling for $a=1$.

conditional mean for the state at a sensor freshly crosses levels. The performance of such a sampling scheme can be analyzed with or without mutual listening of sensor samples. In principle, incorporating packet losses is possible but not transmission delays. This of course adds to the computational burden.

A hybrid sampling scheme based on level-triggered and time triggered sampling lets us stabilize unstable plants using only a finite set. The scheme depends on deterministic sampling when the state is beyond the last level in the finite $\mathcal{L}$. This sort of scheme is needed in practice in order to combat unreliable delivery of packets. However, analyzing and designing such a scheme gets more computationally burdensome.
Extension of the level-triggered scheme to the case of vector state signal is somewhat tricky. On the one hand, levels could be replaced by concentric spherical shells of the form,

$$
|\vec{x}|=l_{i}>0 .
$$

Of course, one could use ellipsoidal shells or other nonsymmetrical and non-concentric shells. But this would differ from a scheme which samples based on threshold crossings of the magnitude of the estimation error signal. The latter scheme would be optimal for the average cost filtering problem when the state signal is scalar and linear.

\section{REFERENCES}

[1] K. J. Åström and B. Bernhardsson, "Comparison of Riemann and Lebesgue sampling for first order stochastic systems," in Proceedings of the 41st IEEE conference on Decision and Control (Las Vegas NV, 2002). IEEE Control Systems Society, 2002, pp. 2011-2016.

[2] I. Karatzas and I.-M. Zamfirescu, "Martingale approach to stochastic control with discretionary stopping," Appl. Math. Optim., vol. 53, no. 2, pp. 163-184, 2006.

[3] I. Karatzas and H. Wang, "Utility maximization with discretionary stopping," SIAM J. Control Optim., vol. 39, no. 1, pp. 306-329 (electronic), 2000.

[4] H. Morimoto, "Variational inequalities for combined control and stopping," SIAM J. Control Optim., vol. 42, no. 2, pp. 686-708 (electronic), 2003.

[5] M. Adès, P. E. Caines, and R. P. Malhamé, "Stochastic optimal control under Poisson-distributed observations," IEEE Trans. Automat. Control, vol. 45, no. 1, pp. 3-13, 2000.

[6] D. Hristu-Varsakelis and P. R. Kumar, "Interrupt-based feedback control over a shared communication medium," in Proceedings of the 41st IEEE conference on Decision and Control and European Control Conference (Las Vegas, 2002). IEEE Control Systems Society, 2002, pp. 3223-3228.

[7] W. Wu and A. Arapostathis, "Optimal control of Markovian systems with observations cost: Models and LQG controls," in Proceedings of American Control Conference, Portland, 2005, pp. 294-299.

[8] V. S. Borkar and P. Varaiya, "Finite chain approximation for a continuous stochastic control problem," IEEE Trans. Automat. Control, vol. 26, no. 2, pp. 466-470, 1981.

[9] O. Hernández-Lerma, Adaptive Markov Control Processes, ser. Applied Mathematical Sciences. New York: Springer-Verlag, 1989, vol. 79.

[10] D. P. Bertsekas, Dynamic Programming and Optimal Control. Vol. II, 2nd ed. Athena Scientific, Belmont, MA, 2001.

[11] B. Øksendal, Stochastic Differential Equations, 6th ed., ser. Universitext. Berlin: Springer-Verlag, 2003, an introduction with applications.

[12] V. S. Borkar, "Controlled Markov chains and stochastic networks," SIAM J. Control Optim., vol. 21, no. 4, pp. 652-666, 1983. 